\title{
Larva migrans cutánea tras un viaje al Caribe
}

\author{
Lisset García-Fernández y María Calderón
}

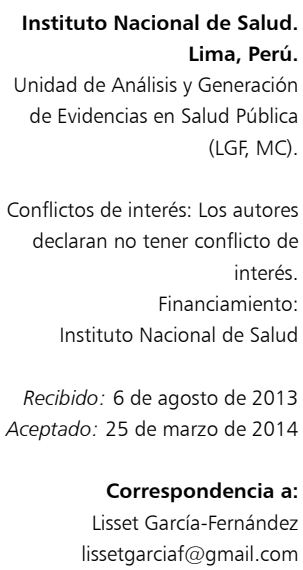

Correspondencia a: Lisset García-Fernández lissetgarciaf@gmail.com

\section{Introducción}

L a larva migrans cutánea es una parasitosis ocasionada principalmente por las larvas de Ancylostoma braziliense y Ancylostoma caninum. Los perros y gatos son los hospederos definitivos, los que depositan sus heces infestadas con huevos contaminando la arena de las playas. Las larvas posteriormente eclosionan por la humedad y el calor. Los seres humanos son afectados por esta enfermedad al tener contacto directo con la arena, como ocurre al caminar con los pies descalzos ${ }^{1-4}$.

El ingreso de las larvas a la piel ocasiona lesiones serpiginosas, eritematosas, levemente solevantadas ${ }^{5,6}$. El diagnóstico de larva migrans cutánea es clínico, dado que las lesiones son muy características. En algunas ocasiones se pueden observar flictenas, edema de la extremidad afectada o puede complicarse con una sobreinfección bacteriana secundaria al grataje $\mathrm{e}^{2,7}$. Incluso pueden simular otras patologías como herpes zoster ${ }^{8}$. El tiempo de presentación de las lesiones es entre uno a cinco días, sin embargo, se han comunicado casos de hasta varios meses tras la exposición. El sitio más frecuente de aparición de las lesiones son los miembros inferiores ${ }^{1,4}$.

En Perú, larva migrans es una entidad poco frecuente $\mathrm{e}^{6,8,9}$. Se presenta el siguiente caso clínico como diagnóstico diferencial entre las enfermedades del viajero ${ }^{10-12}$.

\section{Caso clínico}

Mujer de 27 años de edad, peruana, procedente de Lima, consultó por múltiples lesiones en la piel del pie derecho, pruriginosas, de 10 días de evolución. Posteriormente se asoció edema de la extremidad y malestar general, dos días previos a la evaluación. Como antece- dente, la paciente había visitado Tulum (Riviera Maya, México) durante dos días, en que caminó descalza en la arena y practicó buceo, además de haber sufrido múltiples picaduras por mosquitos en las extremidades inferiores. Había retornado a Lima dos semanas antes de consultar.

Al examen físico, la paciente presentaba múltiples lesiones serpiginosas, solevantadas, eritematosas, confluentes, de uno a tres centímetros de longitud y de uno a dos milímetros de espesor, en el dorso y $2^{\circ}, 3^{\circ}$ y $4^{\circ}$ ortejos del pie derecho. Además, en los dedos se observaban pequeñas vesículas pruriginosas (Figura 1).

Se realizó el diagnóstico clínico de larva migrans cutánea y se indicó tratamiento con ivermectina $6 \mathrm{mg} /$ $\mathrm{ml}$, solución oral en gotas, a dosis de $200 \mu \mathrm{g} / \mathrm{kg}$ de peso, durante dos días y reposo. Presentó un episodio de fiebre, de $38^{\circ} \mathrm{C}$ axilar. Las lesiones empezaron a remitir a partir del tercer día después del tratamiento (Figuras 2 y 3 ).

\section{Discusión}

Esta parasitosis es endémica en las costas del sudeste Atlántico de Norteamérica, el golfo de México, el Caribe y las costas de Uruguay. También se ha reportado en algunas zonas de África, Australia y sudeste de Asia².

El caso presentado corresponde a una larva migrans cutánea, tanto por la clínica como por la epidemiología. El diagnóstico diferencial se planteó con larva currens, causada por otros parásitos intestinales como Strongyloides spp.; sin embargo, éstas se presentan en la zona perineal y migran más rápidamente, lo cual no fue observado en este caso. Entre otros diagnósticos diferenciales se puede plantear escabiosis, loiasis, miasis, esquistosomiasis, tinea corporis y dermatitis de contacto; los que fueron descartados por los antecedentes y las características 
de las lesiones ${ }^{2}$. Para la confirmación del diagnóstico, en algunos casos, se ha utilizado la biopsia cutánea ${ }^{2,6,8}$. Sin embargo, este procedimiento no está recomendado ya que en la mayoría de los casos no se encuentra la larva en la muestra y puede ocasionar daños innecesarios al paciente.

Aunque las lesiones suelen ser autolimitadas y remiten en 4 a 8 semanas, se recomienda dar tratamiento con el fin de acortar la duración del cuadro clínico. Los fármacos indicados para el tratamiento son ivermectina $200 \mu \mathrm{g} / \mathrm{kg}$, por uno a dos días, con tasas de curación entre 81 y $100 \%$ y albendazol 400 a $800 \mathrm{mg}$ al día, durante 3 a 5 días, con una curación entre 46 y 100\%. Anteriormente se recomendaba el uso de tiabendazol, sin embargo, debido a su baja eficacia no se indica para esta patología. También se puede usar tratamiento tópico como tiabendazol, en solución o ungüento, al 10 ó 15\% y crioterapia, los cuales tienen escasa respuesta ${ }^{13}$. En el caso reportado se empleó ivermectina, con lo cual se vio una mejoría rápida de las lesiones.

Esta patología debe tenerse en cuenta en pacientes con el antecedente de haber estado en zonas endémicas y que retornen con lesiones de este tipo. Sin embargo, existen casos reportados en Perú en los cuales no se encontró el antecedente epidemiológico de viajes a zonas endémicas ${ }^{6,8,9}$. Así mismo, en un estudio realizado en Chile para evaluar la presencia de agentes causantes de larva migrans cutánea en muestras de heces caninas, se encontró la presencia de larvas de ancylostomideos en 9,5\% de las muestras, en 8 de las 13 ciudades incluidas ${ }^{14}$. Esto puede indicar que algunos países de Sudamérica, a pesar de no ser endémicos de esta parasitosis, pueden tener algunas zonas con riesgo de transmisión de larva migrans cutánea.

Es importante tener en cuenta que las lesiones cutáneas por larva migrans son una de las consultas más frecuentes en medicina del viajero $^{10-12,15}$; así en el presente caso, tanto las lesiones de la piel, como el antecedente de haber estado en una zona endémica ayudaron al diagnóstico de larva migrans cutánea.

Es importante que los médicos encargados de consejería a los pacientes que viajen a zonas endémicas de esta enfermedad, recomienden medidas preventivas como el uso de calzado adecuado para caminar en las playas. Por otro lado, las medidas de salud pública que se recomiendan en las zonas endémicas son el tratamiento antiparasitario a los perros y gatos, así como la prohibición del ingreso de estos animales a las áreas de bañistas, para así impedir la transmisión de esta parasitosis ${ }^{13}$.

\section{Resumen}

La larva migrans cutánea es una parasitosis ocasionada por las larvas de Ancylostoma braziliense y Ancylostoma caninum, cuyo vehículo es la arena de playas infestadas. Los perros y gatos son los hospederos definitivos. Esta parasitosis es endémica en las costas del Caribe, África, Australia y Asia. Presentamos el caso clínico de una mujer de 27 años de edad, quien tras haber realizado un viaje al Caribe mexicano y haber estado en contacto con arena de playa, presentó unas lesiones en el pie derecho compatibles con larva migrans cutánea. El diagnóstico fue clínico. Recibió tratamiento con ivermectina oral, con buena respuesta.

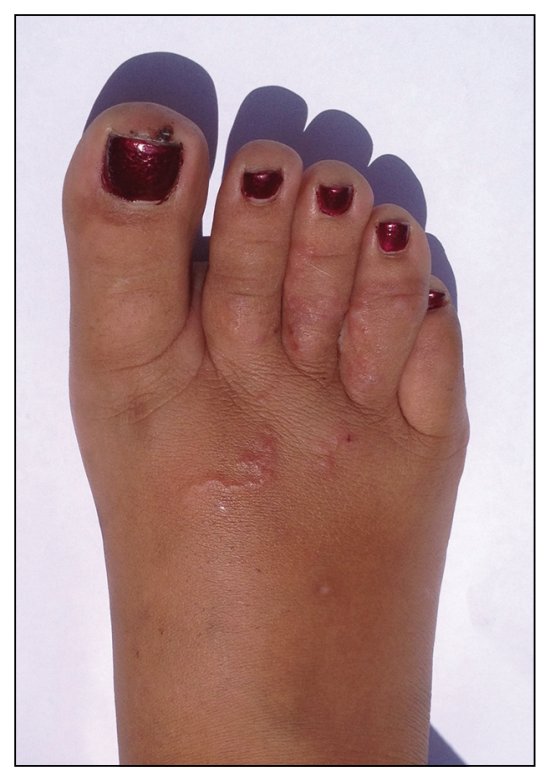

Figura 1. Lesiones serpiginosas, eritematosas y confluyentes en pie derecho al momento del examen.

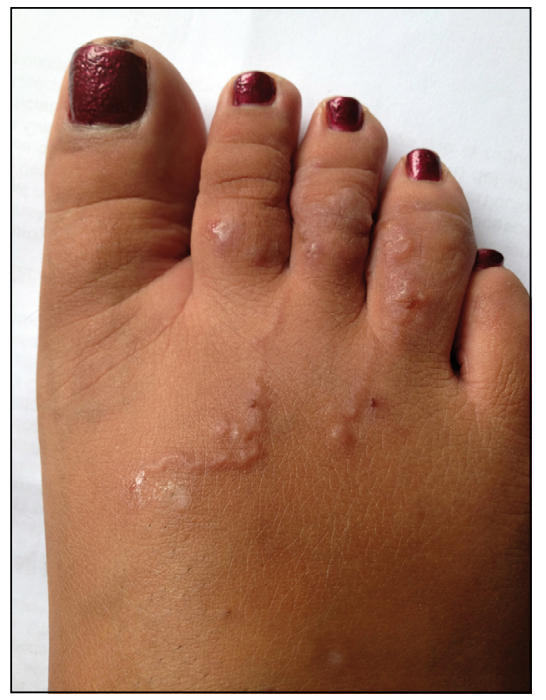

Figura 2. Evolución de las lesiones al tercer día de tratamiento.

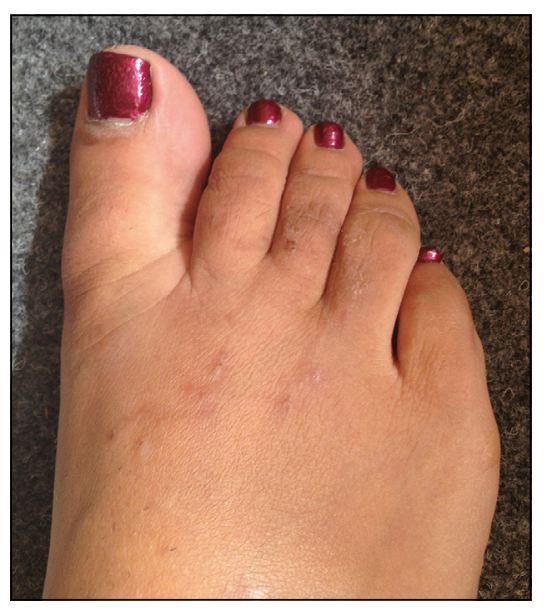

Figura 3. Remisión de las lesiones al séptimo día post-tratamiento. 


\section{Referencias bibliográficas}

1.- Hochedez P, Caumes E. Hookworm-related cutaneous larva migrans. J Travel Med 2007; 14: 326-33.

2.- Heukelbach J, Feldmeier H. Epidemiological and clinical characteristics of hookworm-related cutaneous larva migrans. Lancet Infect Dis 2008; 8: 302-9.

3.- Bowman D D, Montgomery S P, Zajac A M, Eberhard M L, Kazacos K R. Hookworms of dogs and cats as agents of cutaneous larva migrans. Trends Parasitol 2010; 26: 162-7.

4.- Centers for Disease Control and Prevention. Chapter 3: Infectious Diseases Related To Travel-Cutaneous Larva Migrans. Brunette GW, editor. CDC Health Inf Int Travel 2012 Yellow Book. 1st ed. New York: Oxford University Press, USA; 2012. pag: 152-3.

5.- Bava J, González L G, Seley C M, López G P, Troncoso A. A case report of cutaneous larva migrans in Argentina. Asian Pac J Trop Biomed 2011; 1: 81-2.

6.- Escalante E, Rosas N. Larva migrans cutánea. Dermatol Peru 2000; 10: 53-5.

7.- Bordel M T, Sánchez J, Santos J C. Giant blister due to cutaneous larva migrans. Actas Dermosifiliogr 2008; 99: 744-5.

8.- Galarza C, Ramos W, Guere P, Gutiérrez E, Tello M. Larva migrans cutánea simulando herpes zoster. Dermatol Perú 2009; 19: 58-61.

9.- Legua P, Guerra J, Bussalleu A. Larva migrans cutánea en Lima. Reporte de 3 casos. Rev Med Hered 1990; 1: 47-50.

10.- Gautret P, Cramer J P, Field V, Caumes E, Jensenius M, Gkrania-Klotsas E, et al. Infectious diseases among travellers and migrants in Europe, Euro Trav Net 2010. Euro Surveill 2012; 17 (26).

11.- Herbinger K H, Siess C, Nothdurft H D, von Sonnenburg F, Löscher T. Skin disorders among travellers returning from tropical and non-tropical countries consulting a travel medicine clinic. Trop Med Int Health 2011; 16: 1457-64.

12.- Field V, Gautret P, Schlagenhauf P, Burchard G D, Caumes E, Jensenius M, et al. Travel and migration associated infectious diseases morbidity in Europe, 2008. BMC Infect Dis 2010; 10: 330.

13.- Caumes E. Treatment of cutaneous larva migrans. Clin Infect Dis 2000; 30: 811-4.

14.- Mercado R, Ueta M T, Castillo D, Muñoz V, Schenone H. Exposure to larva migrans syndromes in squares and public parks of cities in Chile. Rev Saúde Pública 2004; 38 : 729-31.

15.- Freedman D O, Weld L H, Kozarsky P E, Fisk T, Robins R, von Sonnenburg F, et al. Spectrum of disease and relation to place of exposure among ill returned travelers. N Engl J Med 2006; 354: 119-30. 